\title{
OPEN Quantifying genetic heterogeneity between continental populations for human height and body mass index
}

\author{
Jing Guo ${ }^{1,2}$, Andrew Bakshi ${ }^{3,4}$, Ying Wang ${ }^{1}$, Longda Jiang ${ }^{1}$, Loic Yengo ${ }^{1}$, \\ Michael E. Goddard ${ }^{5,6}$, Peter M. Visscher ${ }^{1,9}$ \& Jian Yang ${ }^{1,7,8,9 凶}$
}

Genome-wide association studies (GWAS) in samples of European ancestry have identified thousands of genetic variants associated with complex traits in humans. However, it remains largely unclear whether these associations can be used in non-European populations. Here, we seek to quantify the proportion of genetic variation for a complex trait shared between continental populations. We estimated the between-population correlation of genetic effects at all SNPs $\left(r_{g}\right)$ or genomewide significant SNPs $\left(r_{g(G W S)}\right)$ for height and body mass index (BMI) in samples of European (EUR; $n=49,839$ ) and African (AFR; $n=17,426)$ ancestry. The $\hat{r}_{g}$ between EUR and AFR was 0.75 (s.e. $=0.035$ ) for height and 0.68 (s.e. $=0.062$ ) for BMI, and the corresponding $\hat{r}_{g(G W S)}$ was 0.82 (s.e. $=0.030$ ) for height and 0.87 (s.e. $=0.064$ ) for BMI, suggesting that a large proportion of GWAS findings discovered in Europeans are likely applicable to non-Europeans for height and BMI. There was no evidence that $\hat{r}_{g}$ differs in SNP groups with different levels of between-population difference in allele frequency or linkage disequilibrium, which, however, can be due to the lack of power.

Most traits and common diseases in humans are complex because they are influenced by many genetic variants as well as environmental factors ${ }^{1,2}$. Genome-wide association studies (GWASs) have discovered $>70,000$ genetic variants associated with human complex traits and diseases ${ }^{3,4}$. However, most GWASs have been heavily biased toward samples of European (EUR) ancestry ( $\sim 79 \%$ of the GWAS participants are of EUR descent $)^{5}$. Progress has been made in recent years in uncovering the genetic architecture of traits and diseases in a broader range of populations ${ }^{6-11}$. Given the population genetic differentiation among worldwide populations $\mathrm{s}^{5,12-15}$, the extent to which the associations discovered in EUR populations can be used in non-EUR such as Africans (AFR) and Asians remains unclear. Genetic correlation $\left(r_{g}\right)$ is the correlation between the additive genetic values of two traits in a population ${ }^{16}$. However, by definition, we cannot observe the trait in AFR and EUR in the same individuals. Therefore, $r_{g}$ is better defined by the correlation between the additive effects of causal variants in the two populations. $r_{g}$ can be less than 1 due to genotype by environment interactions if the two populations are in different environments. Unfortunately, not all the causal variants for complex traits are known so we estimate $r_{g}$ based on the correlation between the apparent effects of genetic markers such as SNPs. This can be estimated by using the genomic relationship matrix (GRM) among all the individuals or, if only summary data is available, the correlation between estimated SNP effects ${ }^{13,17-19} . r_{g}$ estimated from SNPs can be less than that based on causal variants if the LD between causal variants and SNPs differs between the populations. Galinsky et al. ${ }^{14}$ estimated this effect using simulation and found it to be small but this conclusion may not apply to rare causal variants.

\footnotetext{
${ }^{1}$ Institute for Molecular Bioscience, The University of Queensland, Brisbane, QLD 4072, Australia. ${ }^{2}$ Human Genetics, Wellcome Sanger Institute, Hinxton CB10 1SA, UK. ${ }^{3}$ Monash Partners Comprehensive Cancer Consortium, Monash Biomedicine Discovery Institute Cancer Program, Prostate Cancer Research Group, Department of Anatomy and Developmental Biology, Monash University, Clayton, VIC 3800, Australia. ${ }^{4}$ Queensland Brain Institute, The University of Queensland, Brisbane, QLD 4072, Australia. ${ }^{5}$ Faculty of Veterinary and Agricultural Science, University of Melbourne, Parkville, VIC, Australia. ${ }^{6}$ Biosciences Research Division, Department of Economic Development, Jobs, Transport and Resources, Bundoora, VIC, Australia. ${ }^{7}$ School of Life Sciences, Westlake University, Hangzhou 310024, Zhejiang, China. ${ }^{8}$ Westlake Laboratory of Life Sciences and Biomedicine, Hangzhou 310024, Zhejiang, China. ${ }^{9}$ These authors jointly supervised this work: Peter M. Visscher and Jian Yang. ${ }^{\square}$ email: jian.yang@westlake.edu.cn
} 


\begin{tabular}{|l|l|l|l|l|}
\hline & $\hat{\boldsymbol{h}}_{\mathrm{EUR}}^{2}($ s.e. $)$ & $\hat{\boldsymbol{h}}_{\mathrm{AFR}}^{2}($ s.e. $)$ & $\hat{\boldsymbol{r}}_{\boldsymbol{g}}$ (s.e) & $\boldsymbol{P}\left(\boldsymbol{r}_{\boldsymbol{g}}=\mathbf{1}\right)$ \\
\hline Height & $0.50(0.0077)$ & $0.39(0.024)$ & $0.75(0.035)$ & $6.8 \times 10^{-13}$ \\
\hline BMI & $0.25(0.0080)$ & $0.22(0.025)$ & $0.68(0.062)$ & $2.1 \times 10^{-7}$ \\
\hline
\end{tabular}

Table 1. Estimated $\hat{r}_{g}$ between EUR and AFR using HapMap3 SNPs based on the ancestry specific GRMs for height and BMI.

Previous trans-ethnic genetic studies have shown that the estimates of $r_{g}$ at common SNPs (e.g., those with minor allele frequencies (MAF) $>0.01$ ) between EUR and East Asian (EAS) populations are high for inflammatory bowel diseases $\left(\hat{r}_{g}=0.76\right.$ with a standard error (s.e.) of 0.04 for Crohn's disease and $\hat{r}_{g}=0.79$ with s.e. $=0.04$ for ulcerative colitis $)^{20}$ and bipolar disorder $\left(\hat{r}_{g}=0.68\right)^{21}$ and modest for rheumatoid arthritis $\left(\hat{r}_{g}=0.46\right.$ with s.e. $=0.06)^{13}$ and major depressive disorder $\left(\hat{r}_{g}=0.33 \text { with a } 95 \% \text { confidence interval }(\mathrm{CI}) \text { of } 0.27-0.39\right)^{22}$. If the between-population $r_{g}$ for a trait estimated from SNPs is not unity, then it is of interest to know whether the between-population genetic heterogeneity differs at SNPs with different levels of between-population difference in allele frequency (i.e., Wright's fixation index ${ }^{23}, F_{\mathrm{ST}}$ ) or $\mathrm{LD}$, and whether the between-population $r_{g}$ estimated from all common SNPs (MAF > 0.01) can be used to measure the correlation of genetic effects between populations at the genome-wide significant SNPs. Answers to these questions are important to inform the design of gene mapping experiments ${ }^{24-28}$, the genetic risk prediction of complex diseases ${ }^{5,29}$ in the future in non-EUR populations and the detection of signatures of natural selection that has resulted in genetic differentiation among worldwide populations. In this study, we focus on estimating the correlation of genetic effects at all SNPs (denoted by $r_{g}$ ) between continental populations using a bivariate GREML analysis ${ }^{30}$ (treating the phenotypes in the two populations as different traits) for two model complex traits, i.e., height and body mass index (BMI). We investigate the influence of the between-population differences in allele frequencies or LD on the between-population genetic heterogeneity. To do this, we first used genome-wide SNP genotype data to estimate $r_{g}$ between AFR and EUR populations for height and BMI. We also estimated the correlation of genetic effects between continental populations at the genome-wide significant SNPs $\left(r_{g(G W S)}\right)$ identified from an EUR GWAS using the bivariate GREML method ${ }^{30}$ or a summary level data-based method $^{31}$. We then examined whether the between-population genetic overlap is enriched (or depleted) at the SNPs with stronger between-population differentiation in allele frequency or LD.

\section{Results}

Genetic correlation $\left(r_{g}\right)$ between worldwide populations for height and BMI. We used GWAS data on 49,839 individuals of EUR ancestry from the UK Biobank (UKB) and 17,426 individuals of AFR ancestry from multiple publicly available datasets including the UKB (Supplementary Fig. 1; Methods). Note that we used only $\sim 50 \mathrm{~K}$ EUR individuals from the UKB for the ease of computation. All the individuals were not related in a sense that the estimated pairwise genetic relatedness was $<0.05$ within a population. The EUR genotype data were imputed by the UKB (version 3) using the Haplotype Reference Consortium (HRC) and UK10K imputation reference panel ${ }^{32}$. We imputed the AFR data to the 1000 Genomes Project (1000G) reference panel (Methods). After quality control (QC), 1,018,256 HapMap3 SNPs with MAF $>0.01$ in both the two data sets were retained for analysis (Methods). We first used the bivariate GREML approach ${ }^{30}$ to estimate $r_{g}$ between populations as well as the SNP-based heritability $\left(h_{\text {SNP }}^{2}\right)$ in each population for height and BMI. It has been shown in Galinsky et al. ${ }^{14}$ that the estimate of $r_{g}$ from a between-population bivariate GREML analysis is equivalent to the correlation of genetic effect at all SNPs. The GRM used in our bivariate GREML analysis was computed using two different strategies: (1) SNP genotypes standardized using allele frequencies estimated from a combined sample of the two populations (denoted as GRM-average); (2) SNP genotypes standardized using allele frequencies estimated from each population specifically (denoted as GRM-specific; Methods). The $\hat{r}_{g}$ based on GRM-specific was 0.75 (s.e. $=0.035)$ for height and $0.68($ s.e. $=0.062)$ for BMI, suggesting strong genetic overlap between EUR and AFR for both height and BMI (Table 1). The $\hat{r}_{g}$ between EUR and AFR for height was very similar to that between EUR and SAS estimated from the UKB data reported in Galinsky et al. $(0.77 \text { with s.e. }=0.26)^{14}$. We did not observe a substantial difference in $\hat{r}_{g}$ between the analyses based on GRM-average (Supplementary Table 1) and GRM-specific (Table 1). The $\hat{h}_{\mathrm{SNP}}^{2}$ in EUR and AFR from the bivariate GREML analysis were 0.50 (s.e. $=0.0077$ ) and 0.39 (s.e. $=0.024)$ for height, and $0.25($ s.e. $=0.0080)$ and $0.22($ s.e. $=0.025)$ for BMI, respectively $($ Table 1$)$, highly consistent with those from the univariate GREML analysis ${ }^{33}$ where the corresponding estimates were 0.50 (s.e. $=0.0078)$ and 0.40 (s.e. $=0.026)$ for height, and 0.25 (s.e. $=0.0080)$ and 0.23 (s.e. $=0.025)$ for BMI. The first 20 principal components (PCs) were fitted in the bivariate GREML analysis to control for potential effects due to population stratification within populations (Methods). The results were almost identical even without adjustment for PCs (Supplementary Table 2). It is of note that the height $\hat{h}_{\text {SNP }}^{2}$ in EUR was significantly larger than that in $\operatorname{AFR}\left(P=1.3 \times 10^{-4}\right)$, which is consistent with the result from a recent study in European-Americans and African-Americans ${ }^{15}$, presumably because the causal variants in non-Europeans, especially those with MAF $<0.01$, were less well tagged by the SNPs on the SNP arrays compared to those in Europeans. Such a difference was much smaller and not statistically significant for BMI $(P=0.35)$, which can be partly explained by that the imperfect tagging is proportional to trait heritability ${ }^{34}$. To further investigate the difference in SNP tagging between populations, we estimated $h_{S N P}^{2}$ in AFR and EUR in a bivariate GREML analysis based on two subsets of HapMap3 SNPs stratified by whether a SNP is included in the Affymetrix Human Origins (AHO) array $(m=185 \mathrm{k})$ or not $(\mathrm{m}=832 \mathrm{k})$. Unlike the result above, there was no significant difference in the estimated 
a

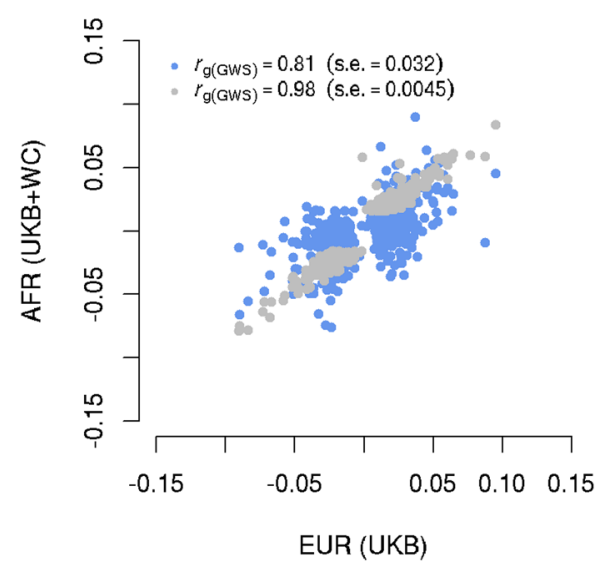

b

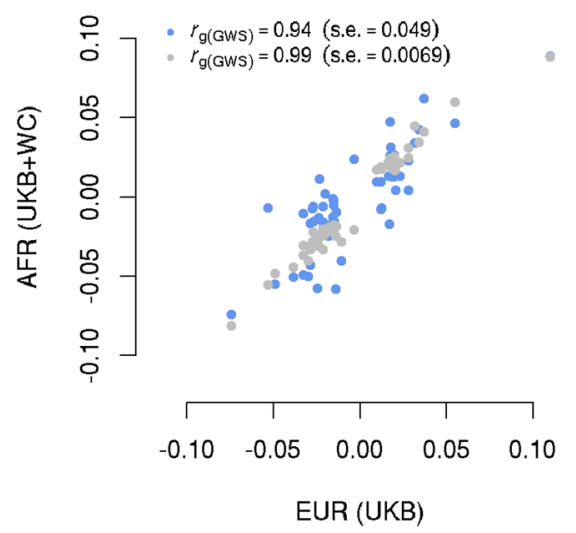

Figure 1. Estimated genetic effect correlation between AFR and EUR for height (a) and BMI (b) at genomewide significant SNPs. The near-independent trait-associated SNPs were discovered in GIANT with their effects re-estimated in our EUR $(n=456,422)$ and AFR $(n=23,355)$ data. The blue dots show a comparison of SNP effects between EUR and AFR and the grey ones show the comparison within EUR (i.e., GIANT vs. EUR-UKB).

height variance explained by the Human Origins SNPs between EUR and AFR $\left(\hat{h}_{S N P(A H O)}^{2}=0.14\right.$ with s.e. $=0.012$ in EUR and $\hat{h}_{S N P(A H O)}^{2}=0.13$ with s.e. $=0.031$ in AFR; $P_{\text {difference }}=0.77$; Supplementary Table 3), suggesting that the observed difference in the $\hat{h}_{S N P}^{2}$ between EUR and AFR using the $\sim 1$ million HapMap3 is possibly attributable to biases in ascertainment of SNPs towards European populations. We further estimated $r_{g}$ between EUR and EAS for BMI by a summary-data-based $r_{g}$ approach ${ }^{13}$ using summary statistics from the GIANT consortium $(n=253,288)^{35}$ and the Biobank Japan project $(\mathrm{BBJ}, n=158,284)^{10}$ (note that the GWAS data with comparable sample size for EAS and the BBJ summary-level data for height were not available to us). The $\hat{r}_{g}$ between EUR and EAS was 0.80 (s.e. $=0.037)$ for BMI, which was also significantly different from $1\left(P=8.36 \times 10^{-8}\right)$, in line with the estimate $(0.75$, s.e. $=0.023)$ from Martin et al. ${ }^{5}$ based on GWAS summary data from the UKB and BBJ.

Correlation of SNP effects between populations at the top associated SNPs. We have quantified above the between-population $r_{g}$ for height and BMI using all HapMap3 SNPs with MAF $>0.01$. The estimates were high but statistically significantly smaller than 1 (Table 1), suggesting there is a between-population genetic heterogeneity for both traits. We know from a previous study that $\hat{r}_{g}$ estimated from all SNPs is close to the estimated causal effect correlation $\left(\hat{\rho}_{b}\right)$ between EUR and SAS ${ }^{14}$. We then sought to ask whether the estimated $r_{g}$ from all SNPs is consistent with that estimated at genome-wide significant SNPs identified in EUR (i.e., $r_{g(G W S)}$ ). We estimated $r_{g(G W S)}$ between EUR and AFR using the recently developed method ${ }^{31}$ that can estimate SNP effect correlation using summary data accounting for errors in the estimated SNP effects (Methods). We used the trait-associated SNPs identified in previous GWAS meta-analyses conducted by the GIANT consortium ${ }^{35,36}$ (with SNP effects re-estimated in our AFR and EUR samples to avoid biases due to the winner's curse; see "Methods"). There were 538 and 57 nearly independent SNPs for height and BMI respectively at $P<5.0 \times 10^{-8}$ selected from clumping analyses $\left(\mathrm{LD} r^{2}\right.$ threshold $=0.01$ and window size $=1 \mathrm{Mb}$ ) of the GIANT summary data (Methods) ${ }^{37}$. To avoid potential bias in estimating $r_{g(G W S)}$ due to remaining LD among these sentinel SNPs, we did an additional round of clumping using a window size of $10 \mathrm{Mb}$ (Methods) and obtained 531 and 56 SNPs for height and BMI respectively. We call these the sentinel SNPs hereafter.

We first estimated $r_{g(G W S)}$ between our EUR sample and GIANT as a "negative control"; the estimate was 0.98 (s.e. $=0.0045$ ) for height and 0.99 (s.e. $=0.0069$ ) for BMI, suggesting no significant differences in SNP effects between the GIANT (a meta-analysis of samples of EUR ancestry) and our sample of EUR participants from the UKB (Fig. 1). We then estimated $r_{g(G W S)}$ between EUR and AFR (SNP effects re-estimated in our samples). We found an estimate of 0.81 (s.e. $=0.032$ ) for height (Fig. 1a) and of 0.94 (s.e. = 0.049) for BMI (Fig. 1b). Since individual-level data were available in our EUR and AFR samples, we performed a bivariate GREML analysis to estimate $r_{g(G W S)}$ only using the sentinel SNPs (Methods); the estimate was 0.82 (s.e. $=0.030$ ) for height and 0.87 (s.e. $=0.064$ ) for BMI, similar to the corresponding estimates using the summary data above. Moreover, summary data-based $\hat{r}_{g(G W S)}$ between EUR (SNP effects re-estimated in this study) and EAS (SNP effects from the BBJ data ${ }^{38}$ ) was 0.90 (s.e. $=0.043$ ) for BMI. All these results suggest that a large proportion of GWAS findings discovered in Europeans are likely replicable in non-Europeans for the two traits (see below for more discussion). In addition, $\hat{r}_{g}$ estimated using all SNPs was largely consistent with $\hat{r}_{g(G W S)}$ for height, but some differences have been observed for BMI (see below for discussion).

Genetic correlation estimated at SNPs stratified by population difference in allele frequency or LD. If there is an effect of the between-population differences in allele frequencies on the between-population genetic heterogeneity for a trait, we hypothesised that the estimate of $r_{g}$ at SNPs with higher $F_{\mathrm{ST}}$ is different 


\begin{tabular}{|l|l|l|l|l|l|l|}
\hline & \multicolumn{5}{|l|}{$\boldsymbol{F}_{S T}$ stratified } & \multicolumn{2}{l|}{ LDCV stratified } \\
\cline { 2 - 7 } & $\hat{\boldsymbol{r}}_{\boldsymbol{g}-\boldsymbol{l}}$ (s.e.) & $\hat{\boldsymbol{r}}_{\boldsymbol{g}-\boldsymbol{h}}$ (s.e.) & $\boldsymbol{P}_{\text {difference }}$ & $\hat{\boldsymbol{r}}_{\boldsymbol{g}-\boldsymbol{l}}($ s.e. $)$ & $\hat{\boldsymbol{r}}_{\boldsymbol{g}-\boldsymbol{h}}$ (s.e.) & $\boldsymbol{P}_{\text {difference }}$ \\
\hline Height & $0.84(0.15)$ & $0.68(0.099)$ & 0.509 & $0.92(0.12)$ & $0.59(0.087)$ & 0.076 \\
\hline BMI & $0.73(0.19)$ & $0.62(0.23)$ & 0.785 & $0.82(0.29)$ & $0.63(0.15)$ & 0.640 \\
\hline
\end{tabular}

Table 2. Difference of the estimated $\hat{r}_{g}$ for EUR-AFR between SNP sets stratified by allele frequency- and LD-matched $F_{S T}$ (and LDCV) for height and BMI respectively. 1 and h indicate the SNP group with lower $F_{S T}$ (or LDCV) and higher $F_{S T}$ (or LDCV), respectively.

from that at SNPs with lower $F_{\mathrm{ST}}$. To test this, we first calculated the $F_{S T}$ values of the HapMap3 SNPs between EUR and AFR. To avoid difference in within-population allele frequency or LD between the two $F_{\mathrm{ST}}$ groups, we divided the SNPs into a large number of bins according to their allele frequencies and LD scores in each population and then stratified the SNPs into two groups with equal number by their $F_{S T}$ values in each MAF-LD bin (Methods). We show that there was no difference in allele frequency or LD score between the two $F_{S T}$ groups after applying this SNP-binning strategy (Supplementary Fig. 2). We performed a two-component bivariate GREML analysis (based on GRM-specific) to estimate $r_{g}$ in each $F_{S T}$ group and found no significant difference in $\hat{r}_{g}$ between the two $F_{S T}$ groups for both traits although the standard errors of $\hat{r}_{g}$ were large (Table 2). Even if our previous study has shown that height increasing alleles are more frequent in EUR than AFR ${ }^{39}$, which might explain the mean difference in height phenotype between EUR and AFR, the result reported here suggests that the population differentiation of frequencies of the height-associated SNPs does not seem to affect the genetic correlation between populations. Nevertheless, it is possible that there is a difference in $r_{g}$ between the two $F_{S T}$ groups but the power of this study is not large enough to detect it.

We applied the same SNP-binning strategy to test whether the estimate of genetic correlation differs when the SNPs are ascertained by difference in LD between populations (Supplementary Fig. 3). We used a metric called LDCV (i.e., coefficient of variation of the LD scores across populations) proposed in a previous study ${ }^{39}$ to measure the differentiation of LD-score between EUR and AFR for each SNP (Methods). We stratified the SNPs into two LDCV groups with no difference in MAF or LD score between the groups in each individual population using the approach described above (Methods; Supplementary Fig. 4) and estimated $r_{g}$ by a twocomponent bivariate GREML analysis. We found no significant difference in the estimate of $\hat{r}_{g}$ between the two LDCV groups (Table 2), which does not support a significant role of LD difference in the between-population genetic heterogeneity at common SNPs but also could be due to the lack of power if the difference in $r_{g}$ between the two LDCV groups is very small.

\section{Discussion}

In this study we showed a substantial genetic overlap at HapMap3 SNPs (MAF $>0.01)$ for height and BMI between EUR and AFR ( $\hat{r}_{g}=0.75$ with s.e. $=0.035$ for height and 0.68 with s.e. $=0.062$ for BMI; Table 1$)$ from a crosspopulation bivariate GREML analysis of individual-level genotype data ${ }^{30}$ and between EUR and EAS $\left(\hat{r}_{g}=0.80\right.$ with s.e. $=0.037$ for BMI) by a summary-data-based approach ${ }^{13}$. All these estimates were significantly smaller than 1 (Table 1), suggesting some genetic heterogeneity between populations for both traits. We then used the recently developed $r_{b}$ approach ${ }^{31}$ that is able to estimate the correlation of SNP effects between populations accounting for estimation errors in estimated SNP effects (Fig. 1), and confirmed the estimates by a bivariate GREML analysis using individual-level data. The bivariate GREML estimate of $r_{g}$ at the sentinel SNPs between EUR and AFR was marginally larger than the estimate for height $\left(\hat{r}_{g(G W S)}=0.82\right.$ with s.e. $=0.030$ vs. $\hat{r}_{g}=0.75$ with s.e. $=0.035 ; P=0.13)$, but the difference was larger for BMI $\left(\hat{r}_{g(G W S)}=0.87\right.$ with s.e. $=0.064$ vs. $\hat{r}_{g}=0.68$ with s.e. $=0.062 ; P=0.032$ ), which may due to a difference in genetic architecture between the two traits and/ or the relatively small number of sentinel SNPs used for BMI. The estimated strong correlation in SNP effect between populations is in line with the finding from previous studies that GWAS results from EUR population are largely consistent with those from non-EUR populations for a certain number of complex traits ${ }^{17,40-45}$. However, the extent to which the EUR-based GWAS findings can be replicated in non-EUR populations can be traitdependent ${ }^{5,22}$. To show this, we estimated $\hat{r}_{g}$ between UKB-EUR $(\mathrm{n}=\sim 450 \mathrm{k})$ and UKB-AFR $(\mathrm{n}=\sim 6,300)$ for 42 additional quantitative traits using Popcorn ${ }^{13}$, a summary data-based approach for estimating cross-population $r_{g}$. The median of the $\hat{r}_{g}$ across the 42 traits was 0.94 , consistent with our conclusion above (Supplementary Fig. 5). We also attempted to quantify the effect of population differentiation in SNP allele frequencies on the betweenpopulation genetic heterogeneity by comparing $\hat{r}_{g}$ estimated from SNPs with higher $F_{S T}$ to that estimated from SNPs with lower $F_{S T}$ but found no significant difference in $\hat{r}_{g}$ between the two $F_{S T}$ groups (Table 2). In addition, it should be noted that differences in SNP effects between populations could reflect the differences in causal effects and/or LD between SNPs and causal variants. Our estimated genetic effect correlation at all SNPs between EUR and AFR for height $\left(\hat{r}_{g}=0.75\right.$ with s.e. $=0.035$; Table 1$)$ was largely consistent with the causal effect correlation between EUR and SAS $\left(\hat{\rho}_{b}=0.78\right.$, s.e. $\left.=0.26\right)$ estimated in a previous study ${ }^{14}$. Although the standard error of $\hat{\rho}_{b}$ is large, the causal effect correlation between EUR and AFR is similar to that between EUR and SAS. Then, the results seem to imply that, on average, the extent to which the difference in SNP effects between populations due to the difference in LD is unlikely to be large for common SNPs. This implication is consistent with our LDCV partitioning analysis which showed no significant difference in $\hat{r}_{g}$ between common SNPs with higher and lower LDCV (Table 2). However, it should be noted that LDCV may differ from the between-population difference in LD between SNPs and causal variants. 
In summary, our study confirmed a large estimate of genetic correlation at common SNPs between worldwide populations for height ${ }^{14}$ and showed a similar level of between-population genetic correlation for BMI. We observed that the estimate of SNP effect correlation at the genome-wide significant SNPs was only marginally larger than the estimate of genetic correlation using all SNPs for height but the difference was more pronounced for BMI. We caution that the difference between $\hat{r}_{g(G W S)}$ and $\hat{r}_{g}$ needs to be quantified in higher precision and the extent to which the between-population genetic heterogeneity for a trait due to differences in allele frequency and LD need to be tested in data sets with larger sample sizes in the future. Moreover, an observed betweenpopulation genetic heterogeneity for a complex trait could also be due to the interactions between genetic (G) and environmental (E) factors. The genotype-by-environment interaction component would be partially eliminated in $r_{g}$ estimation in the study design where two populations differ in genetic ancestry but live in the same environment conditions. We acknowledge that all the conclusions are restricted to common SNPs. The between-population genetic heterogeneity for complex traits at rare variants (or the variants that are rare in one population but common in another) remains to be explored with whole-genome sequence data in large samples ${ }^{46}$. Nevertheless, all our results are consistent with the conclusion that most GWAS findings at common SNPs from EUR populations are largely applicable to non-EUR for height and BMI for variant/gene discovery purposes. However, cautions are required for phenotype (or disease risk) prediction given the limited accuracy of genetic prediction using EUR-based GWAS results in non-EUR populations. As discussed in recent studies ${ }^{5,29}$, a number of genetic and non-genetic factors affect the accuracy of using predictors constructed in EUR populations in non-EUR populations, such as the differences in genetic architecture, allele frequency and LD structure between EUR and non-EUR populations, and the differences in environmental exposures and definitions of clinical phenotypes. By modelling the relative accuracy (RA, relative to the accuracy in populations of same ancestry as the discovery population), Wang et al. quantified how much proportion of the loss of RA using EUR-based PRS in AFR can be explained by the differences in allele frequency and $\mathrm{LD}^{47}$. They found the quantities varied between traits, e.g., $\sim 65 \%$ for height and $\sim 84 \%$ for T2D, reflecting differences in genetic architecture between traits (e.g., heritability, polygenicity and cross-ancestry effect size correlation $)^{47}$. One of the limitations of our study is that African Americans have substantial proportions of European ancestry, and our data do not cover the full diversity of the European and non-European populations. We focus only on the individuals that show similar ancestries with the individuals of European and African ancestries, respectively, in the 1000G (Supplementary Fig. 1). To examine whether the QC step of removing PC outliers is effective for removing AFR individuals with high EUR ancestry, we estimated the percentage of European ancestry using ADMIXTURE in our AFR data before QC, AFR after QC (outlier removal), and unrelated AFR after QC. The results show that our QC steps have effectively removed AFR individuals with high proportions of EUR ancestry (Supplementary Fig. 6; corresponding to the 3 panels in Supplementary Fig. 1). A further QC criterion based on the estimated EUR ancestry (e.g., $>0.1)$ only removed 60 AFR individuals, which did not lead to notable differences in $\hat{h}_{\text {SNP }}^{2}$ and $\hat{r}_{g}$ (e.g., height $\hat{h}_{\text {SNP }}^{2}=0.39$ (s.e. $=0.024)$ in AFR and $\hat{r}_{g}$ between EUR and AFR $=0.75$ (s.e. $\left.=0.035\right)$ using the original QC compared to $\hat{h}_{\mathrm{SNP}}^{2}=0.39($ s.e. $=0.024)$ in AFR and $\hat{r}_{g}=0.75$ (s.e. $\left.=0.035\right)$ with the new QC step for height; Supplementary Table 4). To further demonstrate the effect of the admixture on the $\hat{h}_{\text {SNP }}^{2}$ and $\hat{r}_{g}$, we divided AFR (corresponding to panel 3 in Supplementary Fig. 6) into two groups with higher and lower proportions of European ancestry, respectively ( $n=8,847$ for each group). The EUR data used in bivariate GREML analysis were two random sets $\left(\mathrm{n}=50,000\right.$ for each) from UKB-EUR. We found that compared to the original $\hat{r}_{g}$ between EUR and AFR $\left(\hat{r}_{g}=\right.$ 0.75 with s.e. $=0.035$ for height and $\hat{r}_{g}=0.68$ with s.e. $=0.062$ for BMI), the estimated $\hat{r}_{g}$ appeared to be lower using the AFR samples that have lower proportion of European ancestry $\left(\hat{r}_{g}=0.69\right.$ with s.e. $=0.060$ for height and $\hat{r}_{g}=0.53$ with s.e. $=0.084$ for BMI), and higher using AFR samples with higher proportion of European ancestry $\left(\hat{r}_{g}=0.78\right.$ with s.e. 0.058 for height and $\hat{r}_{g}=0.74$ with s.e. $=0.12$ for BMI; Supplementary Table 5 ). However, none of the difference were statistically significant, which could be due to the limited power of our data.

\section{Methods}

Data. GWAS data of 456,422 individuals of European ancestry were from the UKB (EUR-UKB). GWAS data of 24,077 individuals of African ancestry were from the UKB (AFR-UKB, $n=8230$ ), the Women's Health Initiative (WHI; $n=7480$ ), and the National Heart, Lung, and Blood Institute's Candidate Gene Association Resource (CARe) including ARIC, JHS, CARDIA, CFS and MESA $(n=8367)^{48}$. QC of the UKB SNP genotypes had been conducted by the UKB QC team ${ }^{32}$ and the EUR-UKB data had been imputed to the HRC and UK10K reference panel. For the EUR-UKB imputed data (hard-call genotypes), we filtered out SNPs with missing genotype rate $>0.05, \mathrm{MAF}<0.01$, imputation INFO score $<0.03$ or $P$-value for HWE test $<10^{-6}$. We cleaned the WHI and CARe (AFR-WC) genotype data following the protocol provided by the dbGaP data submitters. We further removed SNPs with SNP call rate $<0.95, \mathrm{MAF}<0.01$ or Hardy-Weinberg Equilibrium (HWE) test $P<0.001$, and removed individuals with sample call rate $<0.9$. We imputed the AFR-UKB and AFR-WC data to the $1000 \mathrm{G}$ using IMPUTE2 ${ }^{49}$, and applied the same filtering thresholds as above to the imputed data. We then combined the cleaned AFR-UKB and AFR-WC as one AFR data set. Since the AFR samples are ancestrally more heterogeneous than the EUR-UKB sample, we removed the AFR individuals whose PC1 or PC2 were more than 6 s.d. away from the mean of the AFR in 1000G in AFR-WC and AFR-UKB separately (the PC-based QC of the EUR-UKB sample was described in a previous study ${ }^{50}$ ). Only the SNPs in common with those in HapMap3 SNPs $(m=\sim 1,018,000)$ were retained for analysis. We used GCTA ${ }^{51}$ to construct the GRM in each population based on all the HapMap3 SNPs and removed one of each pair of individuals with estimated genetic relatedness $>0.05$ in each population (retained 348,501 and 17,693 unrelated individuals in the EUR-UKB and AFR, respectively). These unrelated AFR individuals were a subset of the AFR samples after PC-based QC. The first 20 principal components (PCs) were derived from the GRM in each population. Phenotypes in each population were adjusted for covariates (i.e., age in AFR-WC, and age and assessment centre in EUR-UKB and AFR-UKB) 
in each gender group of each cohort and inverse-normal transformed after removing outliers that were 5 s.d. from the mean for height and 7 s.d. from the mean for BMI (because the phenotype distribution tends to be right skewed for BMI). We choose these two traits because they are two of the most commonly studied quantitative traits and we only have access to the individual-level data of only these two traits in WHI-AFR.

Estimation of $h_{\text {SNP }}^{2}$ and $r_{g}$ using all HapMap3 SNPs. To estimate $h_{\mathrm{SNP}}^{2}$ and cross-population $r_{g}$ for height and BMI, we conducted a bivariate GREML analysis using all HapMap3 SNPs in the unrelated individuals (genetic relatedness $<0.05$ ). For the ease of computation, only 50,000 EUR individuals randomly sampled from the EUR-UKB data were included in the GREML analysis (all the AFR unrelated individuals were included in the analysis). To build the GRM for the bivariate GRM analysis (denoted by GRM-specific), the SNP genotypes were standardized based on the allele frequencies in a specific population (i.e., $\frac{(x-2 p)}{\sqrt{2 p(1-p)}}$ with $x$ being coded as 0 , 1 or 2 and $p$ being the allele frequency in EUR, for example) using GCTA (-sub-popu option) ${ }^{51}$. The bivariate GREML analyses were then performed for height and BMI using the GRM-specific in a combined sample of EUR and AFR. The first 20 PCs generated from the GRM-specific were fitted as covariates in the bivariate GREML to control for population stratification. Only the samples that have both the genotype and phenotype data were included in the bivariate GREML analysis ( $n=49,839$ for EUR and $n=17,426$ for AFR). We also performed the bivariate GREML analyses based on GRMs (and PCs thereof) for which the SNP genotypes were standardized using the allele frequencies computed from the combined sample of EUR and AFR. The bivariate GREML analyses were also performed to estimate $r_{g(G W S)}$ using the GRM-specific built from the sentinel SNPs for both traits. To estimate $r_{g}$ between UKB-EUR and UKB-AFR for the additional 42 quantitative traits, we did GWAS for $\sim 6300$ individuals in UKB-AFR across traits using fastGWA ${ }^{52}$. The GWAS summary data for UKBEUR $\left(\mathrm{n}=\sim 450 \mathrm{k}\right.$ ) have been published in a previous study ${ }^{52}$ and are publicly available (see URLs).

To compare the difference in $\hat{r}_{g}$ between SNP groups with higher and lower $F_{S T}$, we computed $F_{S T}$ between EUR and AFR for each SNP in GCTA (-fst option) ${ }^{51}$. We first split the SNPs into 125 bins according to their MAF in EUR and 125 bins based on the frequencies of the same alleles in AFR ( $125^{\star} 125$ frequency bins in total). We next split each frequency bin into $4 \mathrm{LD}$ bins according to LD scores of the $\mathrm{SNPs}^{34}$ in EUR and 4 bins based on LD scores in AFR. We thereby obtained $250,000\left(125^{\star} 125^{\star} 4^{\star} 4\right)$ bins in total. We then equally divided the SNPs in each bin ( $m=4$ in most bins) into two groups according to the sorted $F_{S T}$ values. There were a small number of bins with only 3 SNPs. For those bins, we randomly allocated 1 or 2 SNPs to the high-F $F_{S T}$ group and the remaining SNPs to the low- $F_{S T}$ group. Finally, we combined the SNPs across all the bins with high and low $F_{S T}$ respectively and computed the GRM-specific for each of the two SNP groups, and fitted the two GRMs jointly in a bivariate GREML analysis to estimate the between-population $r_{g}$ and the population-specific $h_{\mathrm{SNP}}^{2}$ in each $F_{S T}$ group for height and BMI. The first 20 PCs generated from the GRM-specific were fitted as covariates in the GREML analysis. The same strategy was applied to the LDCV stratification based on 250,000 bins including $20^{\star} 20$ frequency bins and $25^{\star} 25 \mathrm{LD}$ bins. The method to compute LDCV has been described elsewhere ${ }^{39}$.

Testing the difference in $\hat{r}_{g}$ between SNP sets. We tested the difference in $\hat{r}_{g}$ between two SNP sets (e.g., the two $F_{\mathrm{ST}^{-}}$-stratified SNP sets described above). We computed the $P$-value for the difference using a $\chi^{2}$ statistic with one degree of freedom, where $\chi^{2}=\frac{\left(\hat{r}_{g 1}-\hat{r}_{g 2}\right)^{2}}{\operatorname{var}\left(\hat{r}_{g 1}-\hat{r}_{g 2}\right)}$ with $\hat{r}_{g 1}$ and $\hat{r}_{g 2}$ representing the estimates of the two SNP sets respectively, and $\operatorname{var}\left(\hat{r}_{g 1}-\hat{r}_{g 2}\right)=\operatorname{var}\left(\hat{r}_{g 1}\right)+\operatorname{var}\left(\hat{r}_{g 2}\right)-2 \operatorname{cov}\left(\hat{r}_{g 1}, \hat{r}_{g 2}\right)$. In the bivariate GREML analysis, $r_{g}$ is defined as $r_{g}=\frac{C_{g\left(p_{1}, p_{2}\right)}}{\sqrt{V_{g\left(p_{1}\right)} V_{g\left(p_{2}\right)}}}$ where $C_{g\left(p_{1}, p_{2}\right)}$ is genetic covariance between populations; $V_{g\left(p_{1}\right)}$ (or $\left.V_{g\left(p_{2}\right)}\right)$ is the genetic variance in a population. The sampling variance of the estimate of $r_{g}$ in a SNP set is

$$
\begin{aligned}
\operatorname{var}\left(\hat{r}_{g}\right)= & r_{g}^{2}\left[\frac{\operatorname{var}\left(\hat{V}_{g\left(p_{1}\right)}\right)}{4 V_{g\left(p_{1}\right)}^{2}}+\frac{\operatorname{var}\left(\hat{V}_{g\left(p_{2}\right)}\right)}{4 V_{g\left(p_{2}\right)}^{2}}+\frac{\operatorname{var}\left(\hat{C}_{g\left(p_{1}, p_{2}\right)}\right)}{C_{g\left(p_{1}, p_{2}\right)}^{2}}+\frac{\operatorname{cov}\left(\hat{V}_{g\left(p_{1}\right)}, \hat{V}_{g\left(p_{2}\right)}\right)}{2 V_{g\left(p_{1}\right)} V_{g\left(p_{2}\right)}}\right. \\
& \left.-\frac{\operatorname{cov}\left(\hat{V}_{g\left(p_{1}\right)}, \hat{C}_{g\left(p_{1}, p_{2}\right)}\right)}{V_{g\left(p_{1}\right)} C_{g\left(p_{1}, p_{2}\right)}}-\frac{\operatorname{cov}\left(\hat{V}_{g\left(p_{2}\right)}, \hat{C}_{g\left(p_{1}, p_{2}\right)}\right)}{V_{g\left(p_{2}\right)} C_{g\left(p_{1}, p_{2}\right)}}\right]
\end{aligned}
$$

The sampling covariance of the estimates of $r_{g}$ between two SNP sets is

$$
\begin{aligned}
& \operatorname{cov}\left(\hat{r}_{g 1}, \hat{r}_{g 2}\right)=r_{g 1} r_{g 2}\left[\frac{\operatorname{cov}\left(\hat{V}_{g\left(p_{1} s_{1}\right)}, \hat{V}_{g\left(p_{1} s_{2}\right)}\right)}{4 V_{g\left(p_{1} s_{1}\right)} V_{g\left(p_{1} s_{2}\right)}}+\frac{\operatorname{cov}\left(\hat{V}_{g\left(p_{1} s_{1}\right)}, \hat{V}_{g\left(p_{2} s_{2}\right)}\right)}{4 V_{g\left(p_{1} s_{1}\right)} V_{g\left(p_{2} s_{2}\right)}}-\frac{\operatorname{cov}\left(\hat{V}_{g\left(p_{1} s_{1}\right)}, \hat{C}_{g\left(p_{1} p_{2} s_{2}\right)}\right)}{2 V_{g\left(p_{1} s_{1}\right)} C_{g\left(p_{1} p_{2} s_{2}\right)}}\right. \\
& +\frac{\operatorname{cov}\left(\hat{V}_{g\left(p_{2} s_{1}\right)}, \hat{V}_{g\left(p_{1} s_{2}\right)}\right)}{4 V_{g\left(p_{2} s_{1}\right)} V_{g\left(p_{1} s_{2}\right)}}+\frac{\operatorname{cov}\left(\hat{V}_{g\left(p_{2} s_{1}\right)}, \hat{V}_{g\left(p_{2} s_{2}\right)}\right)}{4 V_{g\left(p_{2} s_{1}\right)} V_{g\left(p_{2} s_{2}\right)}}-\frac{\operatorname{cov}\left(\hat{V}_{g\left(p_{2} s_{1}\right)}, \hat{C}_{g\left(p_{1} p_{2} s_{2}\right)}\right)}{2 V_{g\left(p_{2} s_{1}\right)} C_{g\left(p_{1} p_{2} s_{2}\right)}} \\
& \left.-\frac{\operatorname{cov}\left(\hat{V}_{g\left(p_{1} s_{2}\right)}, \hat{C}_{\left.g\left(p_{1} p_{2} s_{1}\right)\right)}-\frac{\operatorname{cov}\left(\hat{V}_{g\left(p_{2} s_{2}\right)}, \hat{C}_{g\left(p_{1} p_{2} s_{1}\right)}\right)}{2 V_{g\left(p_{1} s_{2}\right)} C_{g\left(p_{1} p_{2} s_{1}\right)}}+\frac{\operatorname{cov}\left(\hat{C}_{g\left(p_{1} p_{2} s_{1}\right)}, \hat{C}_{g\left(p_{1} p_{2} s_{2}\right)}\right)}{2 V_{g\left(p_{2} s_{2}\right)} C_{g\left(p_{1} p_{2} s_{1}\right)}}\right]}{C_{g\left(p_{1} p_{2} s_{1}\right)} C_{g\left(p_{1} p_{2} s_{2}\right)}}\right]
\end{aligned}
$$

where the subscripts $s_{1}$ (or $s_{2}$ ) represents a SNP set. In practice, the parameters in the equations above can be replaced by their estimates to compute the estimates of $\operatorname{var}\left(\hat{r}_{g}\right)$ and $\operatorname{cov}\left(\hat{r}_{g 1}, \hat{r}_{g 2}\right)$. 
Estimation of SNP effect correlation between populations from GWAS summary data. We obtained the trait-associated SNPs for height and BMI from the GIANT meta-analyses ${ }^{35,36}$. We used the $r_{\mathrm{b}}$ method developed by Qi et al. ${ }^{31}$ to estimate the correlation of SNP effects between populations at the top associated SNPs accounting for sampling errors in the estimated SNP effects. To avoid bias due to 'winner's curse', we re-estimated the SNP effects in our samples (independent from the samples used in the GIANT meta-analysis) using fastGWA ${ }^{52}$. Since fastGWA controls for relatedness ${ }^{52}$, we used all the samples passed QC (including close relatives) for the GWAS analysis ( $n=456,422$ for EUR and 23, 355 for AFR after PC-based QC). The phenotypes were cleaned and normalized using the same strategy described above. The first 20 PCs were included as covariates in the fastGWA analysis to control for population stratification. To get a set of independent SNPs associated with a trait, we did a LD-based clumping analysis in $\operatorname{PLINK}^{37}$ (threshold $P$-value $=5 \times 10^{-8}$, window size $=1 \mathrm{Mb}$ and $\mathrm{LD} r^{2}$ threshold $=0.01$ ). After the clumping analysis, there were 538 and 57 near-independent SNPs associated with height and BMI respectively, which we call sentinel SNPs. To avoid potential bias in $\hat{r}_{g(G W S)}$ due to remaining LD between the sentinel SNPs, we performed an additional round of the clumping analysis with a much larger window size (i.e., $10 \mathrm{Mb}$ ) and obtained 531 and 56 sentinel SNPs for height and BMI respectively. The sampling variance of $\hat{r}_{g(G W S)}$ was computed by a Jackknife resampling process ${ }^{31}$.

URLs. GCTA: http://cnsgenomics.com/software/gcta

PLINK: https://www.cog-genomics.org/plink2

Popcorn: https://github.com/brielin/Popcorn

GWAS summary data for height and BMI in GIANT: https://www.broadinstitute.org/collaboration/giant/ index.php/GIANT_consortium_data_files

GWAS summary data for BMI in Biobank Japan in NBDC Human Database:

https://humandbs.biosciencedbc.jp/en/

UKB consortium: http://www.ukbiobank.ac.uk/

UKB-EUR GWAS summary data: http://fastgwa.info/ukbimp/phenotypes

Affymetrix Human Origins array: http://www.affymetrix.com/support/technical/byproduct.affx?produ $\mathrm{ct}=$ Axiom_GW_HuOrigin

ADMIXTURE: http://dalexander.github.io/admixture/publications.html

\section{Data availability}

See URLs and acknowledgements for GWAS summary data and individual data respectively.

Received: 28 November 2019; Accepted: 22 February 2021

Published online: 04 March 2021

\section{References}

1. Timpson, N. J., Greenwood, C. M. T., Soranzo, N., Lawson, D. J. \& Richards, J. B. Genetic architecture: the shape of the genetic contribution to human traits and disease. Nat. Rev. Genet. 19, 110-124 (2018).

2. Robinson, M. R. et al. Genotype-covariate interaction effects and the heritability of adult body mass index. Nat. Genet. 49, 1174-1181 (2017).

3. Buniello, A. et al. The NHGRI-EBI GWAS Catalog of published genome-wide association studies, targeted arrays and summary statistics 2019. Nucleic Acids Res 47, D1005-D1012 (2019).

4. Visscher, P. M. et al. 10 years of GWAS discovery: Biology, function, and translation. Am. J. Hum. Genet. 101, 5-22 (2017).

5. Martin, A. R. et al. Clinical use of current polygenic risk scores may exacerbate health disparities. Nat. Genet. 51, 584-591 (2019).

6. Martin, A. R. et al. An unexpectedly complex architecture for skin pigmentation in Africans. Cell 171, 1340-1353 (2017).

7. Hoffmann, T.J. et al. A large multiethnic genome-wide association study of prostate cancer identifies novel risk variants and substantial ethnic differences. Cancer Discov. (2015).

8. Rosenberg, N. A. et al. Genome-wide association studies in diverse populations. Nat. Rev. Genet. 11, 356-366 (2010).

9. Hindorff, L. A. et al. Prioritizing diversity in human genomics research. Nat. Rev. Genet. 19, 175-185 (2018).

10. Akiyama, M. et al. Genome-wide association study identifies 112 new loci for body mass index in the Japanese population. Nat. Genet. 49, 1458-1467 (2017).

11. Giri, A. et al. Trans-ethnic association study of blood pressure determinants in over 750,000 individuals. Nat. Genet. 51, 51-62 (2019).

12. Kim, M. S., Patel, K. P., Teng, A. K., Berens, A. J. \& Lachance, J. Genetic disease risks can be misestimated across global populations. Genome Biol. 19, 179 (2018).

13. Brown, B.C., AGENTD Consortium, Ye, C.J., Price, A.L. \& Zaitlen, N. Transethnic genetic-correlation estimates from summary statistics. Am. J. Hum. Genet. 99, 76-88 (2016).

14. Galinsky, K. J. et al. Estimating cross-population genetic correlations of causal effect sizes. Genet. Epidemiol. 43, 180-188 (2019).

15. Veturi, Y. et al. Modeling heterogeneity in the genetic architecture of ethnically diverse groups using random effect interaction models. Genetics 211, 1395-1407 (2019).

16. Falconer, D.S., Mackay, T.F. \& Frankham, R. Introduction to quantitative genetics (4th edn). Trends Genet. 12, 280 (1996).

17. de Candia, T. R. et al. Additive genetic variation in schizophrenia risk is shared by populations of African and European descent. Am. J. Hum. Genet. 93, 463-470 (2013).

18. Yang, J. et al. Genome-wide genetic homogeneity between sexes and populations for human height and body mass index. Hum. Mol. Genet. 24, 7445-7449 (2015).

19. Mancuso, N. et al. The contribution of rare variation to prostate cancer heritability. Nat. Genet. 48, 30-35 (2016).

20. Liu, J. Z. et al. Association analyses identify 38 susceptibility loci for inflammatory bowel disease and highlight shared genetic risk across populations. Nat. Genet. 47, 979-986 (2015).

21. Ikeda, M. et al. A genome-wide association study identifies two novel susceptibility loci and trans population polygenicity associated with bipolar disorder. Mol. Psychiatry 23, 639-649 (2018).

22. Bigdeli, T. B. et al. Genetic effects influencing risk for major depressive disorder in China and Europe. Transl. Psychiatry 7, e1074 (2017).

23. Wright, S. The genetical structure of populations. Ann. Eugen. 15, 323-354 (1951). 
24. Kichaev, G. \& Pasaniuc, B. Leveraging functional-annotation data in trans-ethnic fine-mapping studies. Am. J. Hum. Genet. 97, 260-271 (2015).

25. Ong, R. T., Wang, X., Liu, X. \& Teo, Y. Y. Efficiency of trans-ethnic genome-wide meta-analysis and fine-mapping. Eur. J. Hum. Genet. 20, 1300-1307 (2012).

26. Morris, A. P. Transethnic meta-analysis of genomewide association studies. Genet. Epidemiol. 35, 809-822 (2011).

27. Zaitlen, N., Paşaniuc, B., Gur, T., Ziv, E. \& Halperin, E. Leveraging genetic variability across populations for the identification of causal variants. Am. J. Hum. Genet. 86, 23-33 (2010).

28. Teo, Y. Y., Ong, R. T., Sim, X., Tai, E. S. \& Chia, K. S. Identifying candidate causal variants via trans-population fine-mapping. Genet. Epidemiol. 34, 653-664 (2010).

29. Martin, A. R. et al. Human demographic history impacts genetic risk prediction across diverse populations. Am. J. Hum. Genet. 100, 635-649 (2017).

30. Lee, S. H., Yang, J., Goddard, M. E., Visscher, P. M. \& Wray, N. R. Estimation of pleiotropy between complex diseases using singlenucleotide polymorphism-derived genomic relationships and restricted maximum likelihood. Bioinformatics 28, 2540-2542 (2012).

31. Qi, T. et al. Identifying gene targets for brain-related traits using transcriptomic and methylomic data from blood. Nat. Commun. 9(2018).

32. Bycroft, C. et al. Genome-wide genetic data on 500,000 UK Biobank participants. BioRxiv, 166298 (2017)

33. Yang, J. et al. Common SNPs explain a large proportion of the heritability for human height. Nat. Genet. 42, 565-569 (2010).

34. Yang, J. et al. Genetic variance estimation with imputed variants finds negligible missing heritability for human height and body mass index. Nat. Genet. 47, 1114-1120 (2015).

35. Locke, A. E. et al. Genetic studies of body mass index yield new insights for obesity biology. Nature 518, 197-206 (2015).

36. Wood, A. R. et al. Defining the role of common variation in the genomic and biological architecture of adult human height. Nat. Genet. 46, 1173-1186 (2014).

37. Purcell, S. et al. PLINK: A tool set for whole-genome association and population-based linkage analyses. Am. J. Hum. Genet. 81, 559-575 (2007).

38. Kanai, M. et al. Genetic analysis of quantitative traits in the Japanese population links cell types to complex human diseases. Nat. Genet. 50, 390-400 (2018).

39. Guo, J. et al. Global genetic differentiation of complex traits shaped by natural selection in humans. Nat. Commun. 9, 1865 (2018).

40. Marigorta, U. M. \& Navarro, A. High trans-ethnic replicability of GWAS results implies common causal variants. PLoS Genet. 9, e1003566 (2013).

41. Marigorta, U. M., Rodriguez, J. A., Gibson, G. \& Navarro, A. Replicability and prediction: Lessons and challenges from GWAS. Trends Genet 34, 504-517 (2018).

42. Waters, K. M. et al. Generalizability of associations from prostate cancer genome-wide association studies in multiple populations. Cancer Epidemiol. Biomark. Prev. 18, 1285-1289 (2009).

43. Waters, K.M. et al. Consistent association of type 2 diabetes risk variants found in Europeans in diverse racial and ethnic groups. Plos Genet. 6 (2010).

44. Carlson, C. S. et al. Generalization and dilution of association results from European GWAS in populations of non-European ancestry: The PAGE study. PLoS Biol 11, e1001661 (2013).

45. Fu, J. Y., Festen, E. A. M. \& Wijmenga, C. Multi-ethnic studies in complex traits. Hum. Mol. Genet. 20, R206-R213 (2011).

46. Wainschtein, P. et al. Recovery of trait heritability from whole genome sequence data. bioRxiv, 588020 (2019).

47. Wang, Y. et al. Theoretical and empirical quantification of the accuracy of polygenic scores in ancestry divergent populations. Nat. Commun. 11, 3865 (2020).

48. Musunuru, K. et al. Candidate gene association resource (CARe): Design, methods, and proof of concept. Circ. Cardiovas. Genet. 3, 267-275 (2010).

49. Howie, B. N., Donnelly, P. \& Marchini, J. A flexible and accurate genotype imputation method for the next generation of genomewide association studies. PLoS Genet. 5, e1000529 (2009).

50. Yengo, L. et al. Meta-analysis of genome-wide association studies for height and body mass index in 700,000 individuals of European ancestry. Hum. Mol. Genet. 27, 3641-3649 (2018).

51. Yang, J., Lee, S. H., Goddard, M. E. \& Visscher, P. M. GCTA: A tool for genome-wide complex trait analysis. Am. J. Hum. Genet. 88, 76-82 (2011).

52. Jiang, L. et al. A resource-efficient tool for mixed model association analysis of large-scale data. bioRxiv, 598110 (2019).

\section{Acknowledgements}

This research was supported by the Australian National Health and Medical Research Council (1078037 and 1113400), the Australian Research Council (FT180100186 and FL180100072), the Sylvia \& Charles Viertel Charitable Foundation (Senior Medical Research Fellowship), and the Westlake Education Foundation. This study uses AFR data from the database of Genotypes and Phenotypes (dbGaP) (accession numbers: phs000386 for AFRWHI; AFR-CARe including phs000557.v4.p1, phs000286.v5.p1, phs000613.v1.p2, phs000284.v2.p1, phs000283. v7.p3 for ARIC, JHS, CARDIA, CFS and MESA) and EUR data from the UKB consortium.

\section{Author contributions}

J.Y. and P.M.V. conceived the study. J.Y. and J.G. designed the experiment. J.G. performed statistical analyses under the assistance and guidance from A.B., Y.W., L.J., L.Y., M.E.G., P.M.V. and J.Y., A.B., Y.W., L.J. and L.Y. contributed to data preparation. P.M.V. and J.Y. contributed resources and funding. J.G. and J.Y. wrote the manuscript with the participation of all authors. All authors reviewed and approved the final manuscript.

\section{Competing interests}

The authors declare no competing interests.

\section{Additional information}

Supplementary Information The online version contains supplementary material available at https://doi. org/10.1038/s41598-021-84739-z.

Correspondence and requests for materials should be addressed to J.Y.

Reprints and permissions information is available at www.nature.com/reprints. 
Publisher's note Springer Nature remains neutral with regard to jurisdictional claims in published maps and institutional affiliations.

(c) (i) Open Access This article is licensed under a Creative Commons Attribution 4.0 International License, which permits use, sharing, adaptation, distribution and reproduction in any medium or format, as long as you give appropriate credit to the original author(s) and the source, provide a link to the Creative Commons licence, and indicate if changes were made. The images or other third party material in this article are included in the article's Creative Commons licence, unless indicated otherwise in a credit line to the material. If material is not included in the article's Creative Commons licence and your intended use is not permitted by statutory regulation or exceeds the permitted use, you will need to obtain permission directly from the copyright holder. To view a copy of this licence, visit http://creativecommons.org/licenses/by/4.0/.

(C) The Author(s) 2021 


\section{University Library}

\section{- M M N E R VA A gateway to Melbourne's research publications}

Minerva Access is the Institutional Repository of The University of Melbourne

Author/s:

Guo, J;Bakshi, A;Wang, Y;Jiang, L;Yengo, L;Goddard, ME;Visscher, PM;Yang, J

Title:

Quantifying genetic heterogeneity between continental populations for human height and body mass index

Date:

2021-03-04

\section{Citation:}

Guo, J., Bakshi, A., Wang, Y., Jiang, L., Yengo, L., Goddard, M. E., Visscher, P. M. \& Yang, J. (2021). Quantifying genetic heterogeneity between continental populations for human height and body mass index. SCIENTIFIC REPORTS, 11 (1), https://doi.org/10.1038/ s41598-021-84739-z.

Persistent Link:

http://hdl.handle.net/11343/273132

License:

CC BY 